\title{
Brain-machine interfaces
}

Recent patents related to the translation and communication of neural signals.

\begin{tabular}{|c|c|}
\hline Patent number & Description \\
\hline US 10,092,205 & $\begin{array}{l}\text { Brain-machine interface (BMI) systems or movement-assist systems to aid users with } \\
\text { paraplegia or tetraplegia in ambulation or other movement or in rehabilitation of motor } \\
\text { function after brain injury or neurological disease, such as stroke, Parkinson's disease or } \\
\text { cerebral palsy. The BMI may translate one or more neural signals into a movement type, a } \\
\text { discrete movement or gesture or a series of movements, performed by an actuator. System } \\
\text { and methods of decoding a locomotion-impaired and/or an upper-arm impaired subject's } \\
\text { intent with the BMI may use non-invasive methods to provide the subject the ability to make } \\
\text { the desired motion using an actuator or command a virtual avatar. }\end{array}$ \\
\hline
\end{tabular}

US $9,824,607$

A brain-machine interface for extracting user action intentions within a continuous asynchronous interactive environment. A subliminal stimulus module generates contextually appropriate decision-related stimuli that are unobtrusive to a user. An altered perceptual experience module modifies a user's sensation of the interactive environment on the basis of decision-related stimuli generated from the subliminal stimulus module. A brain monitoring module assesses the user's brain activity in response to the decision-related stimuli and to determine whether an action within the asynchronous interactive environment is intended by the user. Finally, an action is taken based on explicit user input, the user's brain activity in response to the decision-related stimuli, or a combination thereof.

US 9,694,155 A method to induce an immediate psychosomatic change in a patient optimal for a particular medical procedure. The method uses a brain-computer interface integrating a loop of biofeedback and neurofeedback components with interactive, immersive, 3D virtual reality modules using hypnotic or guided-response, learning, placebo and experience-ratification principles.

US 9,539,118 Methods, devices, systems and apparatus, including computer programs encoded on a computer storage medium, for brain-controlled body movement assistance devices. In one aspect, a device includes a brain-controlled body movement assistance device with a braincomputer interface $(\mathrm{BCl})$ component adapted to be mounted to a user, a body movement assistance component operably connected to the $\mathrm{BCl}$ component and adapted to be worn by the user, and a feedback mechanism provided in connection with at least one of the $\mathrm{BCI}$ component and the body movement assistance component.

US 9,373,088 A brain-machine interface for control of prosthetic devices. The interface uses parallel control of a continuous decoder and a discrete action state decoder. In the discrete decoding, we not only learn states affiliated with the task, but also states related to the velocity of the prosthetic device and the engagement of the user. Moreover, we not only learn the distributions of the neural signals in these states, but we also learn the interactions/ transitions between the states, which is crucial to enabling a higher level of performance of the prosthetic device.

US 9,215,298 A method of maintaining an information rate of a brain-computer interface (BCI) system, implanted in a patient's brain, by regulating arousal level in the patient's brain. The method includes selecting a patient with the implanted $\mathrm{BCl}$ device configured to receive neuronal activity from one or more electrodes connected to the patient's brain and to establish a communication channel between the patient and an external device controlled by the patient. A rate of information passage through the communication channel from the $\mathrm{BCl}$ device is measured, and a region of the patient's brain involved in arousal regulation is stimulated in response to said measurement, under conditions effective to adjust the rate of information passing from the $\mathrm{BCl}$ device through the communication channel.

US 9,095,455 A brain-machine interface that incorporates a neural dynamical structure in the control of a prosthetic device to restore motor function and is able to significantly enhance the control performance compared to existing technologies. In one example, a neural dynamical state is inferred from neural observations, which are obtained from a neural implant. In another example, the neural dynamical state can be inferred from both the obtained neural observations and from the kinematics. A controller interfaced with the prosthetic device uses the inferred neural dynamical state as input to the controller to control kinematic variables of the prosthetic device.

\begin{tabular}{lll} 
Assignee & Inventor & Date \\
\hline $\begin{array}{l}\text { University } \\
\text { of Houston }\end{array}$ & $\begin{array}{l}\text { Contreras-Vidal JL, } \\
\text { Prasad S, }\end{array}$ & \\
System & Kilicarslan A, & \\
(Houston, & Bhagat N & \\
TX, USA) & &
\end{tabular}

HRL

Laboratories

(Malibu,

(A, USA)

Bhattacharyya R,

$11 / 21 / 2017$

Uhlenbrock RM,

Payton DW

Panova JS,

Panova JS,

Marcus JD, Marcus JD,

Panova TB Panova TB

$7 / 4 / 2017$

Neurolutions

(Clayton,

Leuthardt EC,

MO, USA)

Love LJ,

Coker R,

Moran DW

The Board

of Trustees

of Stanford

University

(Palo Alto,

(A, USA)

Nuyujukian $\mathrm{P}$,

Kao JC,

Shenoy KV

Cornell

Schiff ND

$12 / 15 / 2015$

University

(Ithaca,

NY, USA)

The Board of Trustees of Stanford University

(Palo Alto,

(A, USA)
Kao JC,

Nuyujukian $P$,

Churchland MM,

Cunningham JP,

Shenoy KV 